\title{
19 Die doppelte Kritik der Philosophischen Anthropologie und die Vieldeutigkeit des theoretischen Anti-Humanismus
}

Aus der Vielzahl von Philosophischen Anthropologien, die ich kenne, überzeugt mich gesamtkonzeptionell nur das Vorgehen von Helmuth Plessner, weil er seine Philosophische Anthropologie klar abgrenzt von anthropologischer Philosophie, d. h. von einer Philosophie, die auf der Grundlage einer anthropologischen Wesensbestimmung des Menschen arbeitet, ohne diese Bestimmung noch in Frage stellen zu können. Mir scheint, dass die meisten Kritiken an Philosophischer Anthropologie auf dieser Verwechselung mit einer anthropologischen Philosophie beruhen. Dies gilt insbesondere für diejenigen Vor-Urteile über sie, die zum sogenannten theoretischen Anti-Humanismus gezählt werden. Im ersten Teil des folgenden Beitrages skizziere ich mein bisheriges Verständnis derjenigen Forderungen, die man „theoretischen Anti-Humanismus“ nennt. Ich hoffe, zumindest einige wichtige seiner Motive $\mathrm{zu}$ erfassen, so dass eine sinnvolle Diskussion möglich wird. Im zweiten Teil stelle ich Plessners doppelte Kritik dar, nämlich seine anthropologische Kritik an der dualistischen Philosophie des Selbstbewusstseins und seine philosophische Kritik an einer abschliessenden anthropologischen Wesensbestimmung des Menschen. Im Durchlaufen beider Teile wird deutlich werden, an welchen Dimensionen des theoretischen Anti-Humanismus in besserer Gestalt festgehalten werden kann und welche seiner Dimensionen sich historisch erübrigt oder gar zu einem Dogma von VorUrteilen verfestigt haben.

\subsection{Verständliche Motive des theoretischen Anti- Humanismus und warum ich dennoch kein theoretischer Anti-Humanist geworden bin}

Bei einem derart großen Thema sei es mir gestattet, dass ich zur Vereinfachung am Beginn einige Stichworte nenne, die bleibende Spuren aus meinen Lektüren von Louis Althusser, Michel Foucault, Jean-François Lyotard und Jacques Derrida benennen. Diese Autoren gehen zum Glück nicht in dem auf, was man ihnen als Label an theoretischem Anti-Humanismus angehängt hat, aber sie gelten doch als Vertreter dieses intellektuellen Markennamens, weshalb ich sie kurz in Erinnerung rufen möchte. Dabei folge ich dem für das Thema des theoretischen Anti-Humanismus einschlägigen Text Fines hominis von Derrida 
(Derrida 1999) darin, dass ich den problemgeschichtlichen Kontext der jeweiligen Lektüren kurz erwähne, um letztere so verständlicher werden zu lassen.

Als ich in der zweiten Hälfte der 1970er Jahre als Student im höheren Semester bzw. als Doktorand Althusser las, war ich froh, dass jemand auf niveauvoll innermarxistische Weise gegen die offizielle Parteiideologie der Neostalinisten darauf bestand, aus Marx eine strenge Wissenschaftlichkeit herauszuholen. Ähnlich wie Marx selbst den wissenschaftlichen Geist der klassischen bürgerlichen Ökonomie gegen den politisch apologetischen Zweck der spätbürgerlichen Ökonomie fortsetzte, versuchte Althusser, Marxens epistemologischen Bruch mit dem Selbstverständnis geschichtlicher Akteure zugunsten der Erkenntnis einer Eigendynamik funktionaler Strukturen freizulegen (siehe Althusser/ Balibar 1972). Es kamen nicht nur ökonomische Verhältnisse und Strukturen, sondern auch Staatsapparate und Anrufungsformen der Bürger zum Vorschein, in denen individuelle und kollektive Subjekte erst produziert werden (Althusser 1977). Ihre formale Gleichstellung als Träger von Freiheitsrechten änderte nicht, sondern reproduzierte ihre reale Ungleichheit in den sozialökonomischen Verhältnissen. Bei allem sympathisierenden Verständnis für Althussers Einsatz gegen die humanistischen Phrasen der bürgerlichen Ideologie, die aus bündnispolitischen Gründen von der neostalinistischen Herrschaftsform übernommen worden waren: Uns in der DDR fehlten - wie in allen Ländern des sowjetischen Imperiums - sogar die formalen Freiheiten und Gleichheiten als Staatsbürger vor dem Gesetz wie auch die Konsumchancen in einer zwar kapitalistischen, aber produktiveren Ökonomie des Westens. Wie der Prager Frühling 1968 in der Tschechoslowakei gezeigt hatte, sprach die humanistische Redeweise vom demokratischen Sozialismus mit einem menschlichen Antlitz doch die überwältigende Mehrheit der Bevölkerung im Osten an und setzte sie für eine nicht nur formale, sondern auch reale Demokratie in Bewegung, die dann aber durch sowjetische Panzer zerschlagen wurde.

Für uns in Osteuropa hatte der junge Marx mit seiner philosophischanthropologischen Kritik am „rohen Kommunismus“, der alle in gemeinsamer Armut $\mathrm{zu}$ Arbeitern eines Staatskonzerns machte, seine kritische Kraft noch nicht verloren. ${ }^{1}$ Althussers Brüche waren selbst noch zu stark in dualistischen

1 Der junge Marx schrieb im Rahmen seiner philosophisch-anthropologischen Kritik an der Entfremdung durch das Privateigentum an Produktionsmitteln über den „rohen Kommunismus“ u. a. Folgendes: „Wie wenig diese Aufhebung des Privateigentums eine wirkliche Aneignung ist, beweist eben die abstrakte Negation der ganzen Welt der Bildung und der Zivilisation, die Rückkehr zur unnatürlichen Einfachheit des armen und bedürfnislosen Menschen, der nicht über das Privateigentum hinaus, sondern noch nicht einmal bei demselben angelangt ist. Die Gemeinschaft ist nur eine Gemeinschaft der Arbeit und die Gleichheit des Salärs, den das 
Hebeln des Entweder-Oder gedacht. Sein Einsatz für die Wissenschaftlichkeit des Marxismus und gegen die Parteiideologie wiederholte lehrreich das alte Problem der Einheit von Theorie und Praxis im Marxismus, indem er beide Seiten dieser Einheit trennte. Diese Trennung provozierte jedoch Althussers Elemente der Selbstkritik (Althusser 1975), wodurch er für sich zur alten Forderung der Einheit von Theorie und Praxis zurückkehrte, für unabhängige und jüngere Leser indessen den Marxismus selbst zur Disposition stellte.

In der ersten Hälfte der 1980er Jahre lasen wir Foucault, angeregt durch den Dramatiker Heiner Müller, mit großem Gewinn, denn er weitete das Thema der epistemologischen Brüche, das Gaston Bachelard eingeführt hatte, enorm und konsequent aus. Zunächst dachte man, er radikalisiere nur das Programm von Marx in den Grundrissen der Kritik der Politischen Ökonomie. Was Hegel und Marx die „verständigen Abstraktionen“ nannten, wie z. B. „der“ Mensch, „die“ Arbeit, „die“ Politik, „die“ Gesellschaft, „die“ Wissenschaft, waren „Realabstraktionen“ (Marx 1953, 21-28), die sich erst in der zweiten Hälfte des 18. Jahrhunderts herausgebildet und bis Max Weber zu den typologischen Aprioris der Moderne entwickelt hatten. Das Ganze der Gesellschaft differenzierte in funktionsspezifische Handlungsbereiche, etwa der Ökonomie, der Politik, des Rechts, der Wissenschaft, Bildung, Kunst etc. aus, wodurch ein offenes Integrationsproblem erneut zum Ganzen der Gesellschaft entsteht. Dieses offene Integrationsproblem moderner Gesellschaften sollte nun im Kampf der Richtungen erneut ökonomisch, politisch oder kulturell gelöst werden, aus marxistischer Sicht aber auf eine gesamtgesellschaftlich reflexive statt allein naturwüchsige Art.

Foucault radikalisierte diese Selbsthistorisierung von Hegel und Marx auf eine originäre Weise, wenn er den anthropologischen Kreis der Moderne mit seinen „empirisch-transzendentalen Dubletten“ (Foucault 1974, 384-390) offen legte. Die Doppelrolle des Menschen bestand darin, dass er einerseits zum empirischen Objekt neuer Positivitäten (der Ökonomie, Biologie, Linguistik) wurde, wodurch eine Analytik seiner Endlichkeit entstand. Andererseits wurde aber der Mensch eben für diese seine Objektivierung auch als eine Ermöglichung in Anspruch genommen, die epistemisch und praktisch-normativ den Status eines Subjekts hatte, das noch nicht objektiviert werden konnte. Zwischen dem Menschen als empirischem Objekt und ihm als dem transzendentalen Subjekt trat die ihm eigene geschichtliche Zeitlichkeit hervor. Er entfremdete sich spontan und unbewusst als Subjekt in objektive Verhältnisse, aus deren Aneignung er

gemeinschaftliche Kapital, die Gemeinschaft als der allgemeine Kapitalist, auszahlt“ (Marx 1973, 535). 
individuell wie kollektiv als ein seiner selbst bewusst werdendes Subjekt erneut hervorgehen sollte. Im Maße der Säkularisierung der religiösen Tradition des Subjekts entstand die Frage, inwiefern der seiner selbst bewusste Subjekt-Status aus der Endlichkeit des Menschen selbst, nämlich seiner Zukunft, ermöglicht werden könne oder doch einer erneut eschatologischen Teilhabe bedürfe. Foucault kritisierte zu Recht an diesem Subjekt-Objekt-Modell, dass es einen Vorrang des Denkens vom Gleichen enthalte, einen Prozess der Entäußerung des unbewussten Subjekts in das ihm ungleiche Objekt und der Wiederaneignung des Subjekts in dem Werden seines Selbstbewusstseins, in dem es mit sich gleich werde, aber auch durch die problematische Angleichung von ihm Fernem und Fremdem: „Denn es ist ein Denken, das nicht mehr zu der niemals beendeten Bildung des Unterschiedes verläuft, sondern zu der stets zu vollziehenden Enthüllung des Gleichen“" (ebd., 409).

In den marxistischen Richtungen lebten nicht nur solche Prozessmodelle vom sich seiner Interessen bewusst werdenden und sie beherrschenden Kollektivsubjekt fort. In ihnen stand damals auch immer die gesellschaftliche bzw. private Aneignung der äußeren Natur im Vordergrund. Über den Doppelcharakter der Arbeit als konkreter und abstrakter, als Gebrauchswert und Wert schaffender Arbeit trat dann der Zusammenhang zwischen dem Verhältnis zur äußeren Natur und den innergesellschaftlichen Verhältnissen hervor. Foucault fokussierte demgegenüber auf die Aneignung der den Menschen eigenen, inneren Natur durch Disziplinierung und Normalisierung der individuellen Körper und der Bevölkerungskörper. Eben diese Strategien der Disziplinierung und Normalisierung von Körpern begegneten uns auch und gerade in den Gesellschaften sowjetischen Typs. Foucault historisierte die marxistische Kritik, indem er ihre Defizite in der historischen Produktion individueller und kollektiver Körper aufdeckte:

Konkret hat sich die Macht zum Leben seit dem 17. Jahrhundert in zwei Hauptformen entwickelt, die keine Gegensätze bilden, sondern eher zwei durch ein Bündel von Zwischenbeziehungen verbundene Pole. Zuerst scheint sich der Pol gebildet zu haben, der um den Körper als Maschine zentriert ist. [...]. [D]ie Machtprozeduren der Disziplinen: politische Anatomie des menschlichen Körpers. Der zweite Pol [...], hat sich um den Gattungskörper zentriert, der von der Mechanik des Lebenden durchkreuzt wird und den biologischen Prozessen zugrunde liegt [...]: Bio-Politik der Bevölkerung. (Foucault 1977, 166)

Umgekehrt fehlte mir bei Foucault eine Philosophie des Lebens, das man als Potential voraussetzen muss, um all diese sozial- und kulturgeschichtlichen Körper-Produktionen in ihrer Selektivität verständlich werden zu lassen. Je klarer einem durch Foucault wurde, wie historisch veränderlich Körper produziert werden können, desto schärfer traten zwei Gegenfragen hervor, zu denen 
Foucault nicht überzeugend Stellung bezog, obgleich er doch Urteile darüber nahelegte, welche Macht- durch Wissens-Formen produktiver als andere Machtformen waren. Erstens: Wie war diese Plastizität von Körpern im Rahmen der Grenzen von anorganischer und organischer Natur möglich? Da Foucault selbst den anthropozentrischen Zirkel der westlichen Moderne offen gelegt hatte, fragte sich, von woher er zu dezentrieren war. Im Unterschied zu Deleuze unternahm Foucault keine Anstrengungen, den Zirkel naturphilosophisch zu dezentrieren. Zweitens: Wie kann man innerhalb der Sozial- und Kulturgeschichte die Grenzen dieser von Foucault aufgedeckten Körper-Techniken und KörperStrategien thematisieren? Sie müssen doch auf die eine oder andere Weise weh tun oder zum Lachen führen! Foucault spielte gerne auf Nietzsches Lachen an, weniger auf dessen großen Leib. An dieser Stelle hatte Foucault auf Artauds Theater der diskurslosen, aber gleichwohl ausdrucksstarken, weil Sprache verdichtenden Leiber verwiesen. „Bei Artaud wird die als Diskurs zurückgewiesene Sprache und in der plastischen Heftigkeit des Zusammenpralls wiederaufgenommene Sprache auf den Schrei, auf den gefolterten Körper, auf die Materialität des Denkens, auf das Fleisch rückverwiesen“ (Foucault 1974, 458).

In der Lektüre Foucaults während der 1980er Jahre hatte ich erstmals den Eindruck, aber nicht die Zeit, sie mit Plessners Philosophischer Anthropologie in Verbindung bringen zu können. Plessner hatte nicht nur in seinen Stufen des Organischen und der Mensch (1928, hier 1975) eine naturphilosophische Dezentrierung des Menschen in der anorganischen und lebenden Natur geleistet. Er war auch in seinem Buch Lachen und Weinen (1941, hier 1982f) den Grenzen des Menschen möglichen Verhaltens nachgegangen, insbesondere im Übergang vom gespielten zum ungespielten Lachen und Weinen. In diesen Erfahrungen tritt die Körper-Leib-Differenz personalen Lebens auffällig hervor. Im Unterschied zu bestimmten Körpern, die personale Lebewesen haben können, indem sie diese nach biowissenschaftlichen und soziokulturellen Maßstäben austauschen, vertreten und ersetzen, leben Personen im Ganzen unersetzbar, unvertretbar und nicht austauschbar als Leib. In dieser Philosophischen Anthropologie wird einerseits durch eine Philosophie der anorganischen und der lebendigen Natur rekonstruiert, in welchen Spielräumen und Spielzeiten anorganische und organische Körper sich verhalten können (sog. vertikaler Vergleich der humanen mit non-humanen Lebensformen). Andererseits wird in ihr thematisiert, welche leiblichen Vollzugsgrenzen soziokulturelle Verkörperungen und Entkörperungen haben (sog. horizontaler Vergleich der humanen Lebensformen untereinander), worauf ich im zweiten Teil zurückkommen werde. An dieser Komplementarität von Plessners und Foucaults Themen und Verfahren, die ich in meinen Publikationen seit 1999 vertrete (Krüger 1999), halte ich noch immer 
fest, insbesondere wenn es um das Thema der Biopolitik geht, die ich aber im Anschluss an Plessners Problematisierung moderner „Lebensmächte“ im weiteren Sinne „Lebenspolitik“ (Krüger 2009a u. 2013a) nenne.

Es ist ganz merkwürdig, wie in der heutigen deutschsprachigen Diskussion im Namen von Foucault gearbeitet wird, ohne sein philosophisches Niveau auch nur zu erwähnen, geschweige denn zu erreichen. Dieser Mangel manifestiert sich u. a. darin, dass noch immer so von seiner Hypothese über das „Ende des Menschen" geschrieben wird, als handele es sich um eine historisch bewährte These und als hätte nicht er selbst sie aus guten Gründen verworfen (siehe Rölli 2015).

\footnotetext{
Wenn ich vom Tod des Menschen spreche, möchte ich allem ein Ende setzen, das dieser Erzeugung des Menschen durch Menschen eine feste Erzeugungsregel, ein wesentliches Ziel vorgeben will. Als ich in der Ordnung der Dinge diesen Tod als etwas dargestellt habe, das sich in unserer Epoche vollzieht, habe ich mich getäuscht. Ich habe zwei Aspekte miteinander verwechselt [...]. Wenn es das Versprechen der Humanwissenschaften war, uns den Menschen zu entdecken, so haben sie es gewiss nicht gehalten; es handelte sich dabei eher um eine allgemeine kulturelle Erfahrung, nämlich die Konstitution einer neuen Subjektivität, vermittelt durch eine Operation, die das menschliche Subjekt auf ein Erkenntnisobjekt reduziert. Der zweite Aspekt, den ich mit dem ersten verwechselt habe, besteht darin, dass die Menschen im Laufe ihrer Geschichte niemals aufgehört haben, sich selbst zu konstruieren, das heißt ihre Subjektivität beständig zu verschieben, sich in einer unendlichen und vielfältigen Serie unterschiedlicher Subjektivitäten zu konstituieren. Diese Serie von Subjektivitäten wird niemals zu einem Ende kommen und uns niemals vor etwas stellen, das ,der Mensch` wäre. (Foucault 1996, 84f.)
}

Man kann in diesen Worten von Foucault aus dem Jahre 1980, d. h. am Beginn seines Spätwerks, eine Verteidigung der Unergründlichkeit des Menschen sehen, um mit Plessner zu sprechen, vor allem aber auch eine Kontinuität in Foucaults Schaffen selbst. Er hatte nicht einfach die Ermöglichung des Objekts „Mensch“ durch das Subjekt „Mensch“ ersetzt, indem er das Subjekt Mensch aus dem Objekt Mensch durch Disziplinierung und Normalisierung erklärte. Vielmehr interessierte er sich für den geschichtlichen Wandel zwischen Subjekt und Objekt, ohne diesen Wandel einseitig als eine Determination der Subjekte durch ihre Verhältnisse als Objekte (sog. Überdeterminierung) aufzufassen. Nicht nur das oben angegebene Zitat von 1980 zeigt, wie sehr Foucault an der Ermöglichung des Objekt-Status durch den Subjekt-Status von Menschen festhielt. Was er den „quasi-transzendentalen Zwillingsbruder“ (Foucault 1974, 393f.) des empirisch objektivierten Menschen genannt hatte, diese geschichtlich für die Zeitgenossen je aktuale, nicht positivierbare Negativität, tauchte bei Hegel in der Tat als die „Nacht“, die den „Menschen“ als ein „reines Selbst“ (Hegel 1987, 172; siehe Krüger 2014a, 296) enthalte, auf. 
Foucaults fortwährendes Interesse am quasi-transzendentalen Status des Menschen trat umso deutlicher erneut in den Versuchen zur Genealogie neuer Formen von Subjektivität in seinem Spätwerk hervor. Ich habe, um die systematische Kontinuität von Foucaults Gesamtwerk zu erfassen, seinen Verweis auf Artauds Theater so gedeutet, als handele es sich in diesem Theater um ein Modell für die Verdichtung der Sprache (eben gerade nicht des Diskurses), die geschichtliche Zeitlichkeit aus dem Ausdruck der Leiber heraus versteht (Krüger 2001, 48f.). ${ }^{2}$ Artauds diskurslose, aber durch Schweigen (Lyotard) und Schrei (Foucault) verdichteten Leiber gingen real- und mediengeschichtlich $u$. a. als die vom Napalm massakrierten Leiber der vietnamesischen Bauern und als die Särge der drogenabhängigen US-Soldaten um die Welt. Aus dem geschichtlichen Umschlagen von Subjekten in Objekte und umgekehrt von Objekten in Subjekte ergab sich zudem auf der philosophisch reflektierenden Ebene das Modell von einem Wettlauf, um eben diese Festsetzung erneut zu öffnen (siehe Krüger 2009a, 105-129).

1987 lernte ich nach seinem ersten offiziellen Vortrag an der HumboldtUniversität zu Berlin dank Frau Christine Pries Monsieur Lyotard persönlich kennen. Ich war nicht nur von seinen Kant-Lektionen (Lyotard 1994) und seiner Interpretation des Enthusiasmus als Geschichtszeichen bei Kant (Lyotard 1988) beeindruckt, sondern vor allem von seinem Buch Der Widerstreit (Lyotard 1987) im Unterschied zu einem „Widerspruch“ überzeugt worden (in Krüger 1993, II. Teil). Zwischen den einander inkommensurablen Diskursarten brach der Widerstreit hervor, weil sie durch keine gemeinsame Metaregel kommensurabel gemacht werden können. Was er die großen „Meistererzählungen“ nannte, beruhte aber gerade auf der überhistorischen Annahme einer solchen Metaregel. Würde sie verwirklicht, geriete man in ein Ende der Geschichte, sei es nun in den Endsieg des ökonomischen Liberalismus, des Kommunismus, des Rassismus oder eines anderen Ismus. In Lyotards Konzeption war Kants Agnostizismus, dem notwendigen Schein einer spekulativ vervollständigenden Vernunft widerstehen zu müssen, mit der sprachpragmatischen Wende des späten Wittgenstein und der Phänomenologie des Sichtbaren und Unsichtbaren (Merleau-Ponty) sehr interessant verbunden worden. Die Parallele zwischen Lyotard und Plessner war offensichtlich. Beide haben Kants theoretischen Agnostizismus, in dem ein Modell der Gewaltenteilung von Vermögen steckt, praktisch transformiert gegen jede Ideologie, die ihre Verwirklichung als das Ende der Geschichte ausgibt - ein falsches und totalitäres Versprechen. Aber auch

2 Für Foucault-Sympathisanten bleibt sein Verweis auf Artauds Theater als einer Verdichtung von Sprache rätselhaft (siehe Gehring 2015, 198-206). 
Lyotard fehlte eine Philosophie der anorganischen und lebendigen Natur, weshalb mir der Zusammenhang seiner Diskursarten mit den Sprachspielen als Lebensformen (Wittgensteins Stichwort), mit Verkörperungen (in Rollen und Technik) und Verleiblichungen (im Habitus, der Mimesis, der sog. zweiten Natur) unklar blieb. Gleichwohl waren wir uns einig darüber, dass Habermas' Der philosophische Diskurs der Moderne (Habermas 1985) eine ganz unglückliche Polemik in die deutsch-französische Diskussion eingebracht hatte, die nicht weiterhalf. Ich freute mich daher sehr über Lyotards Mitarbeit im Beirat der 1993 neugegründeten Deutschen Zeitschrift für Philosophie, dem auch Habermas seitdem angehört.

Schließlich habe ich erst in den 1990er Jahren Derridas Grammatologie (Derrida 1983), Die Stimme und das Phänomen (Derrida 1979) sowie Die Schrift und die Differenz (Derrida 1976) genauer gelesen. Ich fand die Radikalisierung des Vermittlungsgedankens von Hegel über Hegel hinaus und der Potenziale von Charles Sanders Peirces Semiotik sehr anregend, muss allerdings gestehen, dass ich nie an den Primat der Unmittelbarkeit in historischen Prozessen geglaubt hatte, da ich von Hegels Modell der umwegigen List der Vernunft, nicht aber von Husserls Phänomenologie der Wesensschau herkam. Gegen Wesensschauen, die die Realität einklammerten, hatte mich Bertolt Brechts Forderung „Glotzt nicht so romantisch“ skeptisch eingestellt. Interessant war an Derrida, dass er nicht nur den Primat wechselte zugunsten von Vermittlungen wie der Schrift gegenüber der Stimme, sondern auch versuchte, die ganze Ausgangsunterscheidung zwischen der Stimme und der Schrift durch neue Differenzierungsmöglichkeiten zu unterlaufen. So attraktiv dieser Entwurf war, so schwierig ließ er sich ausführen. In Peirces Semiotik waren, anders als es Derrida haben wollte, die vollständigen Zeichen, genannt Drittheiten, nicht nur untereinander zu immer neuen Differenzen zu verflüssigen, sondern auch in Erstheiten in der Wahrnehmung und in Zweitheiten im leiblichen Widerstand der Körper kontextualisiert (Peirce 1983). Dadurch war das Potenzial des Zeichenprozesses, die Semiosis, in Lebensprozesse eingebunden, die zwar auch plastisch waren, denen aber die Leichtigkeit eines Differierens abging, das dem Auseinanderstöbern von Wolken zu gleichen schien. Bei Derrida wurden die Entkopplungen immer nur weiter entkoppelt, vor allem die Signifikanten von den Signifikaten, was angesichts der Analytik der Existenz und Macht bei Foucault auf die Dauer ein lebensfremder Idealismus von erdlosen Zeichen der Zeichen von Zeichen wurde.

Derridas späte, erst 1989 erfolgende Bindung der Dekonstruktion an die Gerechtigkeit verdeutlichte das Problem seiner Experimente, durch alle Verschiebungen der Probleme endlicher Wesen hindurch doch noch urteilsfähig werden 
zu müssen, bevor die historisch Betroffenen verstorben sind. Aber Derrida schien nicht nur das Dekonstruieren der Gerechtigkeit aufzupfropfen, um von ihr eine Urteilsrichtung zu übernehmen, sondern vielmehr die Umkehrung zu behaupten, in deren Namen er selbst sprach: „Die Dekonstruktion ist die Gerechtigkeit“ (Derrida 1991, 30). Diese Bindung der Gerechtigkeit an die Dekonstruktion fand ich vermessen, d. h. kein Zeichen für Souveränität. Souveränität setzte bei Plessner voraus, dass man die Grenzen seines Verhaltens im ungespielten Lachen und Weinen erfährt, es einen leiblich schüttelt bzw. zusammenbrechen lässt. Insofern man, aus solcher Grenzerfahrung kommend, seine erfahrenen Grenzen bejahen kann, werde man souverän, statt sich - sie überspringend - aus Revanche die Rolle Gottes anzumaßen (siehe Krüger 1999, 161164). Derridas Buch über die Politik der Freundschaft (Derrida 2000) war schon ein gestischer Rückfall in eine Imitation Hegels, nämlich in dem Sinne, dass alle anderen Kanonautoren so behandelt wurden, als ob sie nur Vorläufer zu dem Meister selbst gewesen wären. Wer für diese Verbiegung nicht taugte, wurde in diesem Kanon der Selbst-Aufpfropfung nicht erwähnt, weshalb z. B. alle amerikanischen Pragmatisten fehlten. Ausgerechnet der Autor, der früher selbst so viel Bedeutsames zur Dezentrierung geschrieben hatte, war sich nun selbst zu seinem allergrößten Zentrum geworden. Auch in dem Tier, das ich also bin (Derrida 2010; siehe dazu im vorliegenden Buch 23. Kapitel) brach ein Narzissmus aus, der jede Beschäftigung mit den glücklichen, weil sich um die Krone der Schöpfung oder Evolution nicht mehr kümmernden Verhaltenswissenschaften der Gegenwart unmöglich machte, aber natürlich Heideggers Zentrismus geißelte, indem er dessen Geste der Überbietung von allen anderen imitierte. Nun, es gab schon 1946 bessere Heidegger-Kritiken, als Hannah Arendt in ihrem Aufsatz Was ist Existenzphilosophie schrieb, Heideggers Sein und Zeit sei eine Philosophie der „absoluten Selbstischkeit“ (Arendt 1990, 37).

Die Herausforderung Foucault war für mich die größte und sie hält noch heute an. Bei Foucault und Lyotard habe ich keine absoluten Wahrheitsansprüche vernommen; bei Althusser, was seinen Klassenkampf in der Theorie angeht, und bei Derrida, insofern die Dekonstruktion die Gerechtigkeit sein soll, hingegen schon. Derart absolute Wahrheitsansprüche kommen mir wie ein theologisches Bekenntnis vor, das zu jener metaphysischen Onto-Theologie gehört, die zu kritisieren Derrida selbst vorgab. Ich war zu sehr der ostdeutschen Oppositionsbewegung, die sich zwischen dem täglichen westdeutschen Fernsehen und der polnischen Gegengesellschaft von Solidarnosc ausgebildet hatte, verpflichtet, als dass ich der Versuchung hätte folgen können, am Ende erneut das eigene Selbst für das Absolute halten zu müssen. Die Losung dieser Oppositionsbewegung war mit Rosa Luxemburg: Unsere Freiheit ist die Freiheit der Anders- 
denkenden. Sie passte zu diesem Niemandsland der Mauer, auf deren Seiten sich die Nuklearsprengköpfe der Weltmächte gegenüberstanden. Wenn sich irgendetwas aus dem deutschen und europäischen Bürgerkrieg in seinen heissen und kalten Formen inmitten dieser Waffenstarre lernen ließ, dann war es diese Einladung zum Neuanfang an alle 1989.

Was könnte nun aber die genannten französischen Autoren unter dem Label des „theoretischen Anti-Humanismus“ miteinander verbinden? Wenn damit gemeint ist, dass das Philosophieren theoretisch und methodisch frei bleiben soll von dem Zwang, ein bestimmtes Selbstverständnis des Menschen, mithin ein Vor-Urteil zu bestätigen, dann halte ich theoretischen Anti-Humanismus für eine Selbstverständlichkeit, die aber schwer zu leisten ist, weil Menschen die Bewegung durch Bilder, die hier exemplarisch für Schemata stehen mögen, brauchen. Sie haben keine angeborene, sondern eine zu erwerbende Motorik. Nimmt man ihnen jedes Bild aus der Sorge, es könnte ihnen zum Verhängnis ihrer Fixierung werden, können sie sich überhaupt nicht mehr bewegen. Ohne ein geschichtliches Prozessmodell, in dem man Phasen und Dimensionen zu unterscheiden vermag, in denen es besser wäre, diese und nicht jene Bilder oder überhaupt Bilder zu verwenden, wird man nicht urteilsfähig werden. Wenn man das Philosophieren nicht nur als Vorwand der Kritik an den Vor-Urteilen der Anderen und Fremden missbrauchen möchte, muss es die Kritik der eigenen und nächstliegenden Vor-Urteile beinhalten. Kant hat dafür die Figur der Selbstkritik ins Spiel gebracht. Moderne Philosophie ist seit Kant die Selbstkritik der Vernunft, vor allem auch des notwendigen Scheins, der in den Antinomien der Vernunft entsteht, sobald sie sich spekulativ zum Ganzen aufbläst und fanatisch an die Stelle jeder Empirie setzt. In diesem Sinne ist modernes Philosophieren immer Ideologie-Kritik, d. h. Kritik an der Verabsolutierung eines bestimmten Menschen- und Weltbildes, gewesen. Man stelle sich einmal vor, die Anhänger des ganz Anderen als der Vernunft hätten eine Kant ähnliche Leistung in der Selbstkritik an ihrem Fanatismus zustande gebracht!

Das Problem mit diesem kritischen Verständnis modernen Philosophierens entsteht erst, wenn man seine Verknüpfung mit dem Modell einer kopernikanischen Revolution berücksichtigt. Die Ersetzung des ptolemäischen Weltbildes durch ein kopernikanisches Weltbild leuchtet als Dezentrierung insofern ein, als in ihrem physikalischen Resultat die Erde nicht mehr im Zentrum, sondern an der Peripherie der Welt lag. Folgte man dieser physikalischen Revolution wortwörtlich, landete man bei einem archimedischen Punkt in fernen Galaxien, also dort, wo einmal Gott sein sollte und von dem aus die Erde nun als ein nicht minder kleiner Punkt zu berechnen war. In der Übernahme dieser Perspektive des sich in der Unendlichkeit verlierenden archimedischen Punkts bestand seit 
der Neuzeit die eine Variante der Selbstermächtigung des Menschen, die Hannah Arendt die des homo faber nannte (Arendt 1981b, 36. u. 37. Kap.). Aber Kants Transformation dieses Modells besagte philosophisch das gerade Gegenteil, nämlich die Zentrierung auf die Anschauungsformen und Kategorien des transzendentalen Subjekts. Die Ermöglichung der inhaltlichen De-Zentrierung erfolgte durch eine formale Zentrierung, die das transzendentale Subjekt dualistisch abspaltete und zum Konstitutionsgrund der Welt erhob. Man könnte also theoretischen Anti-Humanismus näher als eine Kritik genau daran, an der Begründung der Philosophie durch Vermögen eines dualistisch abgespaltenen Subjekt-Zentrums verstehen, was sowohl individuelle als auch kollektive Subjekt-Zentren betrifft.

Dazu passt, dass Derridas Kritik in Fines Hominis 1968 auf existenzialistische, personalistische und marxistische Subjektphilosophien abzielte: „So definiert war der Humanismus oder Anthropologismus in jener Epoche eine Art gemeinsamer Boden der christlichen oder atheistischen Existentialismen, der spiritualistischen oder nicht-spiritualistischen Wertphilosophie, der rechten oder linken Personalismen, des Marxismus von klassischem Stil““ (Derrida 1999, 139). Man fragt sich beim Lesen dieses Textes nur, warum das Thema des Humanismus, das in der Renaissance nicht an ein Cartesianisches Subjekt gebunden war und in den amerikanischen Pragmatismen der ersten Hälfte des 20. Jahrhunderts eben von einem solchen Konstitutionsgrund erneut befreit worden war, quasi zwanghaft mit der Subjektphilosophie identifiziert werden musste. Gleich der folgende Satz, der mit einem kurzschlüssigen „Und“ fortsetzt, offenbart einen unbedingten Willen zur politischen Provokation: „Und wenn man Anhaltspunkte auf dem Feld politischer Ideologie aufsucht, so war der Anthropologismus der unbemerkte und unwidersprochene Gemeinplatz des Marxismus, des sozialdemokratischen und des christlich-demokratischen Diskurses“ (ebd., 139).

Diese zwanghafte Fehlidentifikation von Humanismus mit Subjektphilosophie wirkt umso weniger überzeugend, als sie am Ende des Textes in das alte Versprechen einer einmal mehr neuen kopernikanischen Revolution führt, sowohl von innen als auch von außen: Es gehe um zwei „Formen der Dekonstruktion“, die es zu „verweben“ und zu „verknüpfen“ (Derrida 1999, 155) gelte:

a. den Ausgang und die Dekonstruktion zu versuchen, ohne den Standort zu wechseln, durch die Wiederholung des Impliziten in den grundlegenden Begriffen und in der ursprünglichen Problematik, durch die Verwendung der Instrumente und Steine, die sich im Haus, das heißt auch in der Sprache, vorfinden, gegen eben dies Gebäude. [...] b. einen Wechsel des Standortes zu beschließen, auf diskontinuierliche und plötzliche Weise, durch ein brutales Sich-außen-Einrichten und durch die Affirmation absoluten Bruchs und absoluter Differenz. (Ebd., 155f.) 
Aber diese Strategie von Dekonstruktion setzt die westliche Moderne als eine Serie kopernikanischer Revolutionen nur fort. Sie konserviert das Avantgardesein-Müssen, indem sie es inhaltlich verschiebt. Immer muss ein neuer Standpunkt, sei es archimedisch von außen im Namen neuer Objektivität, sei es von innen im Namen neuer Subjektivität, als das Unbedingte eingenommen werden, das alle anderen Standpunkte $\mathrm{zu}$ bedingen und so $\mathrm{zu}$ entwerten verspricht, damit die westliche Moderne weiter etwas zu verwerten hat. Hannah Arendt hatte beide Diagnosen, die die Jahrhunderte der westlichen Moderne seit der Neuzeit durchlaufen, kurz und bündig bereits 1958 die vom „Selbstverlust“ oder der „Selbstentfremdung“ und die vom „Weltverlust“ oder der „Weltentfremdung“ (Arendt 1981b, 265, 325) genannt. Für sie bestand indessen das Problem weniger in der Dichotomie zwischen der Innen- und Außenwelt, sondern in deren Ermöglichung durch eine - in ihrer Pluralität - öffentlich teilbare Mitwelt (ebd., 219, 224, 234, 247, 251, 304). Fiel diese öffentlich-plurale Mitwelt aus, zerfiel die Welt in Außen und Innen, in denen dann die Kopernikanischen Revolutionen wie in einer Serie das gemeinsame moderne Leben dualistisch aushebelten.

Man verwechsele diejenige Schein-Kritik, die die westliche Moderne als eine kopernikanische Serie immer neuer Objektivismen und Subjektivismen konserviert, also $\mathrm{zu}$ ihr als Motor gehört, nicht mit denjenigen Kritiken, die wie Hannah Arendts Einsatz auf eine gemeinsame politische Handlungsmacht aus der Pluralität einer öffentlich teilbaren Mitwelt ausgerichtet sind. Zu dieser Art und Weise von Kritik gehört Plessners Buch Die verspätete Nation. Über die Verführbarkeit bürgerlichen Geistes (1935/59, hier 1974). Das Modell des SubjektZentrums als der Ermöglichung einer Dezentrierung sei vom Marxismus im Namen der Gesellschaft, vom Existenzialismus im Namen des Individuums, von Nietzsche im Namen des Übermenschen, von der Geschichtswissenschaft im Namen des Historismus gegen die Gesellschaft, vom Darwinismus im Namen der Evolution gegen den Marxismus und Historismus, schließlich von der Rassentheorie gegen alle anderen gewendet worden (siehe Plessner 1982e, 9.-12. Kap.). Das Ergebnis war die Auflösung der modernen Gesellschaft in den Bürgerkrieg, Weltkrieg und Zivilisationsbruch der Shoa. Die verschiedenen SubjektZentren ermächtigen sich im Konkurrenzkampf zur Hegemonie über alle anderen und landen so im Dezisionismus der Gewalt als Mittel zu ihrem Herrschaftszweck. Die Vervielfältigung der Kopernikanischen Revolution mit je dualistisch exklusivem Wahrheitsanspruch eines Subjekt-Zentrums gegen alle anderen endet in der Selbstdestruktion der Moderne. Ich bejahe also auch den zweiten möglichen Sinn in dem Ausdruck „theoretischer Anti-Humanismus“, wenn damit Kritiken an den Philosophien eines individuellen und/oder kollektiven 
Subjekts gemeint sind, das sich im Scheine einer vermeintlich kopernikanischen Dezentrierung durch Exklusion zur Weltherrschaft ermächtigt. Die Frage ist nur, ob diejenigen, die unter der Flagge des theoretischen Anti-Humanismus segeln, ihr Versprechen wirklich einlösen können oder doch nur wieder eine neue kopernikanische Revolution ausrufen, die im günstigeren Falle in einer Mode statt in einem Bürgerkrieg verschlissen wird.

Wenn man sich ernsthaft fragt, wie man aus dieser Sackgasse dualistisch abgespaltener Subjekt-Zentren, die alle eine Dezentrierung versprechen, herauszukommen vermag, dann muss man sich wohl fragen, wie man die dualistischen Hebel der Entweder-Oder-Alternativen verlassen kann. Max Schelers Phänomenologie bahnte - im Gegensatz zu der in den Meditationen wieder dualistisch gewordenen Phänomenologie Husserls - einen Zugang zum Leben, indem er die exklusiven Alternativen, das Phänomen müsse entweder materiell oder geistig und entweder physisch oder psychisch sein, methodisch außer Kraft setzte. Umgekehrt: Wenn sich die Phänomene von sich aus als sowohl materiell als auch geistig und als sowohl physisch als auch psychisch zeigen, können sie als lebendige gelten (Scheler 1995, 18f., 39, 42, 74, 79). Leben ist demnach hybrid. Scheler brauchte systematisch noch einen spekulativen Seinsgrund, ein ens a se, während Plessner den Organisationsweisen lebender Körper nach innen und ihren Positionierungsweisen nach außen ins letztlich Unergründliche folgte. Personales Leben heißt, sich in offener Fraglichkeit halten zu können. Seine Rekonstruktion der ontischen und ontologischen Präsuppositionen der modernen Naturwissenschaften, vor allem der Biowissenschaften, legte Lebensprozesse frei, in denen nur unter bestimmten strukturalen und funktionalen Bedingungen auch Bewusstsein und Selbstbewusstsein eine Rolle spielen. Diese Subjekte resultieren aus den Werdensprozessen des Lebens, wenn die Organisations- und Positionsform zentrisch ist. Nicht die Subjekte konstituierten den Lebenskreis, die Umwelt oder Welt, den Lebensprozess im Ganzen, sondern umgekehrt: Die Sphären des Lebens ermöglichen verschiedene Arten und Weisen, in denen sich lebende Körper vor-zentrisch, zentrisch und ex-zentrisch aufführen können. Plessners Untersuchungsverfahren war „quasitranszendental“ (Krüger 2001, 88-93) in dem Sinne, dass er noch immer die philosophische Frage nach den Ermöglichungsstrukturen von Phänomenen stellte. Aber diese Ermöglichungsstrukturen lagen nicht mehr im überhistorischen Bewusstsein eines transzendentalen Subjekts, sondern in den verschiedenen, sowohl a-zentrischen als auch zentrischen wie exzentrischen Arten und Weisen, im Prozess aus ihrer Zukunft heraus zu leben.

Dies bedeutete im politischen Umkehrschluss, dass man personale Lebewesen gerade ihrer Lebensweise beraubt, wenn man sie gegen ihren Lebenspro- 
zess separiert. Plessner verteidigte daher die Grundrechte lebender Personen auf ihre Freiheit in einer modernen Gesellschaft - nicht, weil er darin das Ende der Geschichte sah, sondern den Anfang für einen wertedemokratischen Wettbewerb der Kulturen. „Denn der Begriff des Menschen ist nichts anderes als das ,Mittel', durch welches und in welchem jene wertedemokratische Gleichstellung aller Kulturen in ihrer Rückbeziehung auf einen schöpferischen Lebensgrund vollzogen wird“ (Plessner 1981d, 186). Die Bejahung der Grundrechte und der Wertedemokratie erfolgte historisch, um eine gemeinsame Zukunft zu ermöglichen, die im Zeichen der Unergründlichkeit des Menschen stand. Mir scheint noch immer, dass Plessners Bejahung der Grundrechte und der Wertedemokratie als einer Ermöglichung von gemeinsamer pluraler Zukunft politisch besser ist als Heideggers Anti-Humanismus, der sich nicht von seinem weltgeschichtlichen Verständnis des Nationalsozialismus trennen lässt, wie es inzwischen auch wieder seine „Schwarzen Hefte“ bestätigt haben.

Warum bin ich am Ende kein theoretischer Anti-Humanist geworden? Politischer Anti-Humanist bin ich ohnehin nicht, weil ich mit Plessner und Arendt das Politische von der öffentlich zu teilenden Mitwelt her erfahren habe. Ich bin auch theoretisch kein Anti-Humanist, weil ich das Philosophieren und das Politische nicht abhängig machen kann von diesem oder jenem Humanismusverständnis, von diesem oder jenem Anthropomorphismus, der sich als Anthropologie ausgibt. Wer „Anti“ ist, macht sich abhängig von dem, wogegen er ist. Ich veranschlage die Freiheit des Philosophierens und der öffentlichen Entdeckung des Politischen größer, als sie in irgendeiner Anti-Rolle bestimmt werden können. Mir hat das Dagegen-Sein philosophisch und politisch nie genügt, weil es einen im günstigsten Fall an Gegner, im ungünstigsten Fall an Feinde fesselt, statt selbst mit Anderem und Fremdem als Anderem und Fremdem ein besseres Leben zu beginnen. Die Selbstfestlegung auf das „Anti“ des gerade für human oder up to date Gehaltenen ist mir zu unfrei. Sie erschien und erscheint mir als nur ein weiteres Dogma, durch das man sich schon wieder über das Leben stellt, um es zu beherrschen, statt sich in seiner Hybridität und Offenheit zu halten.

\subsection{Plessners Programm einer anthropologischen Kritik am Dualismus und einer philosophischen Kritik am anthropologischen Monismus}

Im Folgenden möchte ich Plessners Programm der Philosophischen Anthropologie anhand von vier Fragen so skizzieren, wie ich es verstehe und angesichts des theoretischen Anti-Humanismus vertreten kann. 
Erstens: Was ist „Philosophische Anthropologie“ im Unterschied zu „philosophischer Anthropologie“ und „anthropologischer Philosophie“?

Unter „Anthropologie“ wird die Lehre (aus griech.: logos) vom Menschen (griech.: anthropos) verstanden (siehe Landmann 1982). Sie hat insbesondere seit dem 18. und bis ins 20. Jahrhundert $\mathrm{zu}$ einer Vielfalt von erfahrungswissenschaftlichen Anthropologien (biologische, medizinische, geschichtliche, politische, Sozial- und Kultur-Anthropologien bzw. Ethnologien) geführt. Im Unterschied zu diesen Anthropologien beschäftigt sich die philosophische Anthropologie mit dem Wesen des Menschen, das - alle anthropologischen Teilaspekte strukturell integrierend - in der Lebensführung als ganzer vollzogen wird. Seit den 1920er Jahren ist umstritten, ob die philosophische Anthropologie nur eine besondere Disziplin innerhalb der Philosophie darstellt, welche die erfahrungswissenschaftlichen Anthropologien generalisierend integriert, oder ob sie darüber hinaus die Fundierungs- und Begründungsaufgaben der Philosophie selbst übernehmen kann. Der letztere Anspruch wird „Philosophische“ Anthropologie genannt, es wird also „Philosophisch“ groß statt klein geschrieben. Diese terminologische Unterscheidung hat Plessner in seiner Groninger Antrittsvorlesung, 1936 gehalten und 1937 publiziert, eingeführt (Plessner 1983d, 33-51). Man ist ihm darin bis heute gefolgt (Schnädelbach 1983, 269-272; Fischer 2008, 14f.), hat aber den dritten Ausdruck von der ,anthropologischen Philosophie“ weggelassen, der jedoch relational ausschlaggebend ist. Man könnte den Übergang von der innerphilosophischen Subdisziplin ,philosophische Anthropologie“ in die Philosophie „Philosophische Anthropologie“ so verstehen, dass man die allgemeine Integration der erfahrungswissenschaftlichen Anthropologien zum Fundament der Philosophie macht. Genau dies nennt Plessner „,anthropologische Philosophie“ (Plessner 1983d, 36-39; Plessner 1983i, 242-245). Sie nutzt eine allgemein integrierende Anthropologie zur Kritik der Philosophie, und dies heißt vor allem zur Kritik an dem dualistischen Hauptstrom moderner Philosophie (seit Descartes und Kant) und dessen Folgen, etwa neuen Einheitsmythen. Bleibt man aber bei dieser anthropologischen Kritik der Philosophie stehen, schafft man die Philosophie zugunsten des anthropologischen Zirkels ab. Viel wichtiger ist der zweite Schritt, nun umgekehrt die Frage zu stellen, was die Anthropologien praktisch im Leben und in ihrer Forschung voraussetzen, das sie selbst nicht verstehen und erklären können (Krüger 2009a, 4. Kap.; Wunsch 2014, 3.3. u. 4. Kap.). Diese praktischen Voraussetzungen der Anthropologien, die in ihnen in Anspruch genommen werden, nicht aber in ihnen erklärt und verstanden werden können, dürften selbst zur Offenheit der spezifisch personalen Lebensformen gehören. Diese lebensweltlichen Präsuppositionen ermöglichen Anthropologie als eine Leistung im personalen 
Leben. Dadurch werden die Anthropologien rückwirkend in ihren kognitiven und praktischen Geltungsansprüchen wieder einer philosophischen Grenzbestimmung unterzogen. Heute würde man von einer Analyse und Rekonstruktion der lebens- und forschungspraktischen Präsuppositionen anthropologischer Forschungen sprechen.

Es ist diese Doppelbewegung von der anthropologischen Kritik der dualistischen Philosophie zu einer erneut philosophischen Kritik der Anthropologie, die in dem Ausdruck „Philosophische Anthropologie“ gefordert und zumeist verkannt wird. Diese Doppelkritik stellt die Aufgabe einer Zukunft ermöglichenden Überkreuzung dar. Die Philosophische Anthropologie behandelt als Philosophie die Grenzfragen der menschlichen Lebensführung, aber als Anthropologie auf die Themen und Methoden zweier Vergleichsreihen bezogen, die für die dichotomische Moderne konstitutiv sind, also deren immanent ansetzende Kritik erlauben. Es geht um die „horizontale“ und „vertikale“ Vergleichsreihe (Plessner 1975, 32): In der vertikalen Richtung wird die Gattung bzw. Spezies menschlicher Lebewesen mit anderen organischen (pflanzlichen und tierlichen) Lebensformen im Hinblick auf die Frage verglichen, ob die Spezifikation des Menschen im Rahmen der lebendigen Natur hinreichend erfolgen kann oder darüber hinaus durch einen „Wesensunterschied“ (Scheler 1995, 36) fundiert und begründet werden muss. In horizontaler Richtung werden Soziokulturen des homo sapiens sapiens untereinander im Hinblick auf das für die Spe-zifikation menschlichen Daseins wesensnötige Minimum an Möglichkeiten verglichen. Dies erfolgt sowohl historisch unter Einschluss ausgestorbener als auch in der Unterscheidung gegenwärtig lebender Soziokulturen. In der englischen und französischen Literatur wird das zuletzt genannte Vergleichsproblem auch unter dem Namen der Ethnologie diskutiert. Der Zusammenhang der vertikalen und horizontalen Spezifikationsrichtungen des Menschen als Individuum und Gattung wird selbst geschichtlich herausproduziert und bedarf daher einer „politischen Anthropologie“ der „geschichtlichen Weltansicht“ (Plessner 1981d, 139-144).

Zweitens: Worin besteht die theoretische Spezifik der Philosophischen Anthropologie?

Beide anthropologischen Vergleichsreihen werden unabhängig voneinander fundiert, wodurch sie sich gegenseitig in Frage stellen und korrigieren können, um Speziesismen (vertikal) und Ethnozentrismen oder Anthropozentrismen (horizontal) aufdecken zu können. Aus dem gleichen Grunde werden beide einem indirekten Frageverfahren unterworfen. Der Philosophische Anthropologe ist nicht direkt der bessere erfahrungswissenschaftliche Anthropologe, son- 
dern untersucht indirekt, was letzterer lebens- und forschungspraktisch in Anspruch nimmt, ohne es selbst erklären und verstehen zu können. Die lebenspraktischen Präsuppositionen kommen aus der Praxis des common sense, die forschungspraktischen aus der Zukunft, also aus der Fortsetzung der Forschung selbst.

Für die naturphilosophische Fundierung der anthropologisch vertikalen Vergleiche besteht die Hypothese der Philosophischen Anthropologie darin, dass für diese Vergleiche in der lebendigen Natur eine exzentrische Positionalität in Anspruch genommen wird. Im Unterschied $\mathrm{zu}$ den „Organisationsformen“, welche die Binnendifferenzierung von Organismen betreffen, sind die Formen der Positionalität Verhaltensweisen von Organismen in ihrer Umwelt. Exzentrische Positionierungen sind nicht nur an eine zentrische Organisationsform, sondern auch an eine zentrische Positionalitätsform gebunden. Es gibt also die Möglichkeit, zwischen dem Zentrum des Organismus und dem Zentrum in den Interaktionen des Organismus mit seiner Umwelt eine funktionale Korrelation herzustellen. Aber über diese zentrische Korrelation hinausgehend können personale Lebewesen außerhalb dieses organischen Zentrums und jenes Verhaltenszentrums in der Umwelt Verhalten bilden. Könnten sie dies nicht, könnten sie auch keine Korrelationen feststellen, sondern säßen in diesen fest.

Personen können sich aber aus einer Welt, insbesondere Mitwelt (Plessner 1975, 302-308) heraus symbolisch perspektivieren und positionieren. Personale Lebewesen stehen mithin vor dem Problem, die Exzentrierung und die Rezentrierung ihres Verhaltens ausbalancieren zu müssen. Ihr Verhalten unterliegt grundgesetzlichen Ambivalenzen, die strukturell aus dem Bruch zwischen physischen, psychischen und geistigen Verhaltensdimensionen hervorgehen, wobei aber dieser Bruch im Vollzug des Verhaltens verschränkt werden muss (ebd., 292f.). Die drei wichtigsten Verhaltensambivalenzen, in denen exzentriert und rezentriert wird, bestehen in einer „natürlichen Künstlichkeit“ (ebd., 309ff.), einer „vermittelten Unmittelbarkeit“ (ebd., 321ff.) und einem „utopischen Standort“ (zwischen Nichtigkeit und Transzendenz, ebd., 341ff.). Diese Ermöglichungsstruktur personalen Lebens werde im Ganzen und als wesentlich unterstellt, wenn die Spezifik der menschlichen im Unterschied zu nonhumanen Lebensformen bestimmt wird, z. B. von Bioanthropologen, medizinischen Anthropologen, Hirnforschern.

Für die geschichtsphilosophische Fundierung (Mitscherlich 2007) der anthropologisch horizontalen Vergleiche besteht die Hypothese der Philosophischen Anthropologie in Folgendem: Das Wesen des Menschen im Ganzen seiner Lebensführung liegt in seiner „Unergründlichkeit“ (Plessner 1981d, 160-165, 181, 202, 222-228; siehe im vorliegenden Band 17. Kapitel), d. h. im homo abscondi- 
tus, der Zukunft ermöglicht. Damit kann dieses Wesen im Ganzen nicht abschließend bestimmt werden. Dies schließt ein, dass es unter Aspekten und Perspektiven sehr wohl bestimmt und bedingt werden kann, sofern es endlich ist, z. B. durch Geisteswissenschaften bzw. Humanwissenschaften. Für diese Bestimmbarkeit hat Plessner eine Theorie des Spielens in und mit soziokulturellen Rollen entworfen, das vom ungespielten Lachen und Weinen begrenzt wird (siehe Krüger 1999, im vorliegenden Band Zweiter Teil). Lebte dieses Wesen praktisch nicht in einer Relation der Unbestimmtheit von Zukunft auf sich hin, hätte es keine Bestimmungsaufgabe mehr vor sich. Es wäre bereits vollständig determiniert. Es würde vielleicht durch Geschichte bedingt, würde aber keine Geschichte mehr machen können. Es hätte mithin keine Zukunft, aus der her es im Unterschied zu seiner Vergangenheit lebt, vergegenwärtigt, vollzieht. Die geistige Zurechenbarkeit geschichtlicher Prozesse auf menschliche Lebewesen bleibt von den Naturkörpern, den soziokulturellen Rollenkörpern und der leiblichen Performativität der Rollenträger begrenzt (Plessner 1981d, 226-228).

Drittens: Wie verfährt die Philosophische Anthropologie methodisch?

Sie modifiziert vier philosophische Methoden in einem Untersuchungsprozess, glaubt also nicht, dass allein eine Methode bereits eine Philosophie ergibt. Sie übernimmt auch nicht aus der Erfahrungswissenschaft Methoden, sondern behandelt deren Selektivität. Sie reformuliert die phänomenologische Methode (von Husserl und Scheler herkommend), die hermeneutische Methode (von Dilthey in der systematischen Interpretation durch Georg Misch genommen), die verhaltenskritische (dialektische Krisen im personalen Verhaltensaufbau rekonstruierende) und die transzendentale (den Ermöglichungsgrund einer personalen Lebensleistung im Ganzen rekonstruierende) Methode. Diese vier Methoden werden in einem Untersuchungsverfahren integriert, um die oben genannten Grundhypothesen und weitere Zwischenhypothesen überprüfen zu können.

a) Es bleibt das methodische Verdienst von Max Scheler, Husserls phänomenologische Methode von der Rückkehr in die transzendentale Bewusstseinsphilosophie befreit zu haben. Um die Begegnung mit und Beschreibung von spezifisch lebendigen Phänomenen $\mathrm{zu}$ ermöglichen, neutralisiert Scheler das phänomenologische Verfahren gegen die dualistische Vorentscheidung, das Phänomen müsse entweder physisch oder psychisch bestimmt werden. Dadurch kann das Phänomen sich von sich aus als gerade und nur in dem Doppelaspekt zwischen Physischem und Psychischem Lebendiges zeigen (Scheler 1995, 17-19, 39, 42). Auf dem Verhaltensniveau von personalen Lebewesen greift phänomenologisch die bereits oben erwähnte Körper-Leib-Differenz. 
b) Spätestens in der Beschreibung des begegnenden Phänomens wird es den habitualisierten und aufmerkenden Erwartungen entsprechend interpretiert. Wie es bedingt, bestimmt und verendlicht wird, hängt davon ab, in welchen Relationen und anhand welcher Horizonte von Unbedingtem, Unbestimmtem und Unendlichem es aufgefasst wird. Die Interpretation des Anwesenden hängt von Abwesendem ab. Es gibt nicht nur ein unmittelbares Verstehen der Oberfläche von lebendigem Ausdruck in der Verhaltensreaktion, sondern auch ein variables Ausdrucksverstehen und symbolische Verständnismöglichkeiten, die sich von der Verhaltensreaktion hier und jetzt abkoppeln (in Präzisierung Diltheys, Plessner 1975, 28-37).

c) Durchläuft man Spektren von Phänomenen und Spektren ihrer Interpretation kommt man zu der Frage, wann und wo, unter welchen Umständen Verhaltenskrisen eintreten. In solchen Krisen ist die Zuordnung zwischen Phänomenbegegnung und seiner im Verhalten angemessenen Interpretation grundsätzlich in Frage gestellt. Es treten Zusammenbrüche des personalen Verhaltens auf. Ein Musterbeispiel für die dialektisch-kritische Methode hat Plessner in seinem Buch über gespieltes und ungespieltes Lachen und Weinen (1941, hier 1982f) vorgelegt. Semiotisch-strukturell hat er sich mit dieser Frage auch in den Fällen beschäftigt, in denen keine personal-funktionale Einheit der symbolischen Integration von Sinnesmodalitäten zu Stande kommt, so in seinen Büchern Einheit der Sinne (1923, hier 1980b) und Anthropologie der Sinne (1970, hier 1980a). Die dialektischen Verhaltenskrisen stellen die Zuordnung zwischen der Frage, die ein Ausdrucksphänomen an uns richtet (a), und der Antwort, die wir ihm im Verhalten geben (b), nochmals in Frage: Wie bauen die kategorialen Einheiten von Anschauungs- und Verständnisweise aufeinander auf?

d) Hat man im Untersuchungsverfahren die Verhaltensspektren phänomenologisch, hermeneutisch und im Hinblick auf Verhaltenskrisen durchlaufen, fragt sich, welche Ermöglichungsstrukturen im Ganzen wesentlich sind. Dies zeigt sich daran, ob die Verhaltenskrisen in neuen Leistungen personalen Lebens überwunden werden können. Souveränität liegt nicht in absoluter Selbstbestimmung und Selbstverwirklichung vor, sondern hebt darin an, sich zu den Grenzen derselben doch lebensbejahend verhalten zu können. Natürlich kann man die methodische Schrittfolge erneut für Korrekturen durchlaufen. Zu der Beurteilung der Untersuchung gehört die selbstkritische Analyse des semiotischen Organons, das man verwendet und durch das man die Verhaltensspektren womöglich nicht angemessen erfasst hat. Gleichwohl ist jetzt die theoretische Ebene der Beurteilung erreicht: Worin bestanden Defizite in physischer, psychischer und geistiger Hinsicht der Verhaltensverschränkung? Unter welchen Aspekten und in welchen Perspektiven tritt die Verunmöglichung perso- 
nalen Verhaltens ein? Welche Über- oder Unterdeterminierungen gab es? Welcher Bezug ergibt sich zu den beiden o. g. Haupthypothesen über welche Vermittlungen?

Viertens: Wie thematisiert die Philosophische Anthropologie das Politische?

Diese Thematisierung geschieht auf zweierlei Weisen, nämlich durch eine gesellschaftliche Öffentlichkeit zivilisatorischen Verhaltens, das im Gegensatz zu Gemeinschaftsformen der Pluralität Rechnung trägt und die Individuen in ihrer Privatheit freihält. Die gesellschaftliche Öffentlichkeit wird jedoch de facto durch radikale Einschränkungen, die für die westliche Moderne charakteristisch sind, reduziert, marginalisiert und womöglich sogar aufgelöst.

Zunächst wird das Politische neu ermöglicht durch das Öffentliche der Gesellschaft im Unterschied zu den familienähnlichen Gemeinschaftsformen oder den Gemeinschaftsformen einer rationalen Leistungsart. Gemeinschaftsformen beruhen darauf, dass ihre Mitglieder dieselben Werte teilen, sei es gefühlt in familienähnlicher Form der Generationenfolge, sei es durch den Beitrag zu geistig geteilten Wertorientierungen (z. B. in einer scientific community). Gemessen an den gemeinschaftlich geteilten Werten gibt es eine klare Bestimmbarkeit des individuellen Verhaltens, in familienähnlicher Form durch persönliche Hierarchien der Liebe, in sachlicher Form durch die Beurteilung bestimmter Leistungen seitens unabhängiger dritter Personen (siehe Plessner 1981b, 45-57). Gemeinschaftsformen sind untereinander inkommensurabel. Die grundgesetzlichen Ambivalenzen in der personalen Verhaltensbildung bedürfen beider Vergemeinschaftungsformen (ebd., 63-76). Diese Ambivalenzen (z. B. im Verschränken von Verhaltenheit und Scham einerseits, Geltungsdrang und Exponiertheit andererseits) wenden sich aber zugleich gegen ihre Auflösung in diese eine und keine andere Vergemeinschaftunsgsform. Stattdessen bedürfen die Individuen auch der Wertferne in der Interaktion mit Anderen und Fremden, die anders und fremd bleiben können, gemessen an bestimmten Gemeinschaftswerten. Gesellschaftsformen bilden gegenüber den Gemeinschaftsformen die Interaktion mit Anderem und Fremdem als Anderem und Fremdem aus. Dafür braucht man eine Öffentlichkeit, die diplomatisch den Umgang von Rollenträgern ermöglicht, welche privat für einen taktvollen Umgang frei bleiben. Die Politik steht damit vor der Aufgabe, durch das Recht die Ansprüche auf Gemeinschaftlichkeit und Gesellschaftlichkeit auszugleichen, um die Vielfalt individuellen Lebens zu ermöglichen (ebd., 115-133). Sie verfehlt ihre Aufgabe, wenn sie in den Dualismus von entweder Kommunitarismus oder Liberalismus führt. 
Die gesellschaftlich öffentliche Ermöglichung neuer Politikformen entspricht der lebensphilosophischen Orientierung an dem oben genannten homo absconditus. Sie gehört zu einer wertepluralen Gesellschaft, die ihre unvermeidlichen Konflikte zivilisiert auszutragen sucht (siehe Plessner 1981d, 161-164, 185-191, 201-204). Sie kann aber auf zwei Weisen eingeschränkt werden, die in der westlichen Moderne üblich geworden sind. Dann wird entweder die Politik einer anthropologischen Wesensdefinition des Menschen unterstellt, wie dies nicht nur in den nationalsozialistischen und bolschewistischen Gemeinschaftsideologien der Fall war (der Mensch als Rassen- oder Klassenwesen). Es gab schon seit Hobbes pessimistische und seit Rousseau optimistische Anthropologien, auf deren Basis entsprechende Verfassungen gefordert und erlassen wurden. Wie immer erfahrungsgesättigt diese Anthropologien bereits gewesen sein mochten, sie wurden soziokulturell durch ihre umkämpfte Verfassungsgeschichte $\mathrm{zu}$ einem funktional historischen Apriori künftiger Erfahrungen des sozialen Zusammenlebens in der westlichen Moderne, heute z. B. als homo oeconomicus. Oder das Politische als die Ermöglichung von empirischer Politik wird seiner eigenen Autonomie überlassen: Dann wird es weder einer gegenüber positiven Absolutismen skeptischen Lebensphilosophie noch einer (material oder formal) bestimmten Anthropologie unterworfen. Stattdessen wird es an der Intensivierung von Freund-Feind-Verhältnissen ausgerichtet, welche die Unheimlichkeit in der conditio humana interessenbedingt für die Durchsetzung klarer Entweder-Oder-Alternativen durch Politiken der Angst ausnutzt (ebd., 192-200). Der Kampf um das Primat in der Menschenfrage, ob sie nämlich anthropologisch definitiv beantwortet oder der Politik in ihrer Autonomie überlassen oder philosophisch begründet in gesellschaftlicher Öffentlichkeit offen gehalten wird, ist selbst die in der Moderne entscheidende Strukturpolitik, in der Lebensmacht gewonnen und verloren, Lebenspolitik entgrenzt und begrenzt wird. 
